\title{
Implementation of Energy Efficiency Based on Time Scheduling to Improve Network Lifetime in Wireless Body Area Network (WBAN)
}

\author{
Subono, M. UdinHarun Al Rasyid, I Gede Puja Astawa \\ Electronic Engineering Polytechnic Institute of Surabaya \\ Jalan Raya ITS Surabaya 60111, Indonesia \\ E-mail: subono@pasca.student.pens.ac.id, \{udinharun,puja\}@pens.ac.id
}

\begin{abstract}
ZigBee applications of IEEE 802.15.4 Wireless Sensor Network (WSN) with Low Rate Wireless Personal Area Network (LR-WPAN) can be integrated with e-health technology Wireless Body Area Network (WBAN). WBAN are small size and can communicate quickly making it easier for people to obtain information accurately.WBAN has a variety of functions that can help human life. It can be used in the e-health, military and sports. WBAN has the potential to be the future of wireless communication solutions. WBAN use battery as its primary power source. WBAN has limited energy and must be able to save energy consumption in order to operate for a long time. In this study, we propose a method of time scheduling called cycle sleep period (CSP) as WBAN solutions to save energy and improve energy efficiency. The CSP method is implemented in the real hardware testbed using sensor e-health includes temperature body and current sensor. We compared the performance of CSP method with duty cycle management (DCM) time scheduling-based and without using time scheduling.From the measurement results, our proposed idea has decreasingenergy consumption.
\end{abstract}

Keywords: WSN, LR-WPAN, WBAN, e-health, Time Scheduling

\section{INTRODUCTION}

Low rate wireless personal area network (LR-WPAN) with IEEE 802.15.4 is a standardized specification of physical and medium access control (MAC) layer for short range and employ low-power communication devices with low computing capability[1]. Wireless sensor network (WSN) with LR-WPAN has recently been applied to wide application areas, such as home automation [2], industrial automation [3], agriculture [4], military [5], healthcare [6],etc. The healthcare application of wireless body area network (WBAN) can be usedas a wearable and implantable sensor device. The wearable medical sensor devices are temperature measurement, respiration monitor, heart rate monitor, pulse oximeter Sp02, blood pressure monitor, $\mathrm{pH}$ monitor, glucose sensor, etc. The implantable medical sensor devices are those that are inserted inside human body [7]. WBAN is one of the efficient 
technologies for healthcare system. Integrated WBAN and WSN can be taken biomedical signal at the regular interval from the people who live in the dormitory or in the yard rest home. The biomedical signal can be transferred to the Nursing Center [8].

WBAN sizes small and can communicate for another device to PAN coordinator quickly and accurately. WBAN with star and tree topology has three function devices, such as end device with reduce function device (RFD) and PAN coordinator with full function device (FFD). Xbee 802.15.4 series 2 is device for communication from WBAN and generally operates with limited battery power. Therefore, WBAN continuously consumes low energy and LRWPAN achieves longer operation time.

The end device node WBAN uses limited energy. This paper examines the energy efficiency with time scheduling method. In this paper, we compared the performance of cycle sleep period (CSP) method with duty cycle management (DCM) time scheduling-based and without using time scheduling. The length of the active period and sleep period in a sensor can be change.

\section{RELATED WORKS}

Several research related to energy efficiency have been conducted. Lee et al. [9] proposed superframe adjustment and beacon transmission scheme (SABTS) by assigning the accurate values of beacon order and superframe order for network nodes in order to decrease packet transmitted collisions. The analytical based on markov chain model and simulation of SABTS method show that SABTS perform better in term of probability of successful transmission, throughput, and energy consumption.

In paper [10-11], the authors proposed the adaptation of duty cycle management (DCM) to handle energy efficiency. Kim et al. [10] designed dynamic duty cycle (DDC) method to handle energy efficiency. DDC was varying the sleep period according to packet traffic and its priority. The simulation result of DDC by using NS2 achieved improvement in terms of latency and energy efficiency.Rasouli et al. [11] proposed adaptive duty cycle (ADCA) algorithm to maximize the use of network traffic for automatically adjusting the duty cycle for minimum energy. ADCA selects the superframe order value for each superframe according to idle time, throughput of contention access period (CAP), and queue state of node. The OMNET++ simulation result of ADCA show superiority in terms of throughput and energy consumption than standard IEEE 802.15.4.

Deepak et al. [12] analyzed the energy efficiency of Wireless Body Area Networks (WBAN) in scheduled accesss mode. The authors analyzed the impact of superframe size, number of uploads per superframe, payload size, and acknowledgement policy by using the mathematical numerical. Huque et al. [13] proposed an energy efficient adaptive routing in wireless body area network (EAR-BAN). EAR-BAN form cluster and body node coordinator (BNC) selects cluster head $(\mathrm{CH})$ depending on their available energy. BNC 
will process data transmission of body node (BN) depending on its avaiable energy and the distance between BN and BNC.

Paper [14] and [15], proposed wakeup radio (WUR) method to analyze the energy efficiency of WBAN. Karvonen et al. [14] and Petajajarvi et al. [15] proposed generic wakeup radio (WUR) based MAC protocol (GWRMAC). GWR-MAC includes a bidirectional wake-up procedure, sourceinitiated and sink-initiated. The authors also described analytical model which is used to explore the energy efficiency of GWR-MAC. GWR-MAC protocol is applyed in assumption scenario human body monitoring. The numerical analysis shows that the energy efficiency of GWR-MAC is better than that of DCM in light detected events per year. However, the energy efficiency of GWR-MAC is lower than that of DCM in high detected events per year.

\section{ORIGINALITY}

WBAN is a new technology for wireless communication. WBAN has ability to solve a variety of problems, especially for e-health. WBAN can monitor the health condition of the dynamic patient position. Every time the end device nodes send bio-medic data to the PAN coordinator and server. End device node in general consumes low power because the battery as the main power source, so that communication between nodes in the network requested energy consumption as low as possible to operate with a long time. In this paper, we implement real hardware testbed for e-health WBAN includes temperature sensor to monitor vital sign of body and current sensor to analyze the energy consumption of battery. As described in related works section, there are several research have been conducted related to energy efficiency. None of the proposed method in related works have implemented in real hardware testbed. Most of the proposed methods in related works were analyzed by using NS2 simulation, OMNET++ simulation, and mathematical analysis.

This paper proposes time scheduling method named TBS and CSP parameters which are implemented in real e-health hardware testbed.TBS and the CSP is the time period that is setup on the Xbee 802.15.4 pro series 2 . In the WBAN, pro 802.15.4 Xbee series 2 is used as a transmitting and receiving data between the end device and the PAN coordinator. Microcontroller arduinouno on the end device function receives data from the e-health shield sensors, ACS712 current sensor, and battery voltage. Microcontroller arduinouno then process the data and transferred to the Xbee 802.15.4 pro series 2 to be transmitted towards the PAN coordinator.We analyze the comparison of energy consumption among proposed method CSP, DCM, and without using time scheduling. 


\section{SYSTEM DESIGN}

WBAN consists of multiple nodes; each node has the sensing, communicating, and computing ability. The amount of power consumed by WBAN depends on many variables: network topology, number of nodes, etc. Node in the WBAN, in general, consumes low power because the primary power source of WBAN is battery. Basically, the network consists of logical and physical topology. Logicaltopology is more complex than physical topology. Logical topology describes data flow in the network. Physical topology describes placement, device location, and cable installation.

Logical network topology is applied in the WBAN to observe energy consumption, energy efficiency, and transmission data from end device to PAN coordinator. WBAN has the characteristics of low power and low rate communication. Classification for point to point communication betweenPAN coordinator and end devicesis describes in Figure 1. The Figure 1 consists of PAN coordinator which has full function device (FFD), end device which has reduce function device (RFD), Body temperature sensor, currentACS712, and voltage sensor. End Device collects the data from the sensor. Dataare processed in the end device and sent to the PAN coordinator with Xbeewireless communication 2.4 GHz. Data from PAN coordinator will besent with serial data communication to be processed in the server.Figure 2 describes the system design of PAN coordinator. In this figure, PAN coordinator consists of Xbee802.15.4 Pro 2 device for data receiver from end device. Data are sent to the server for further processing.

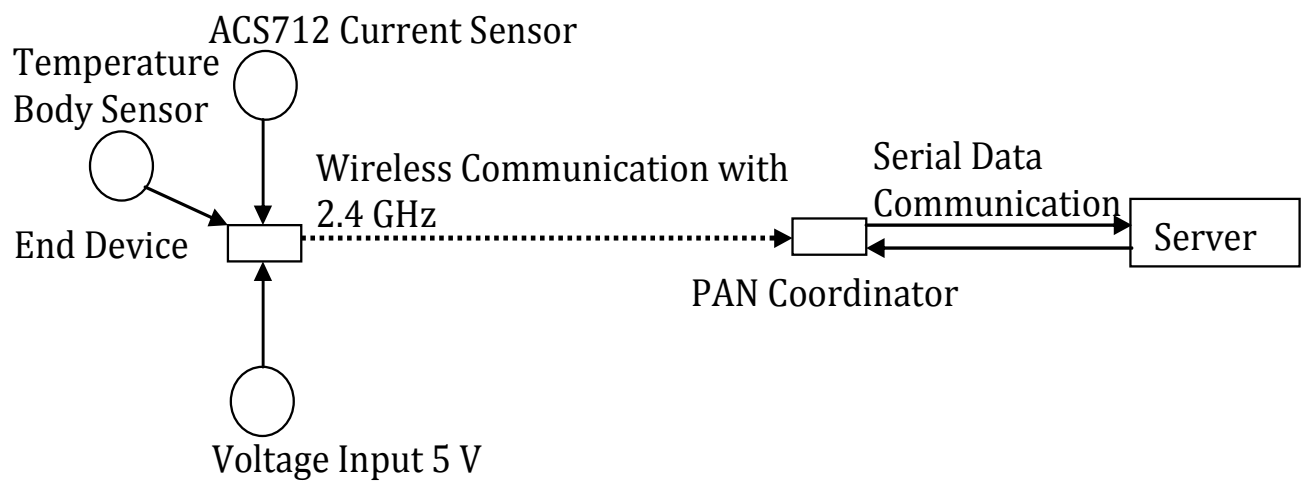

Figure 1. System diagram

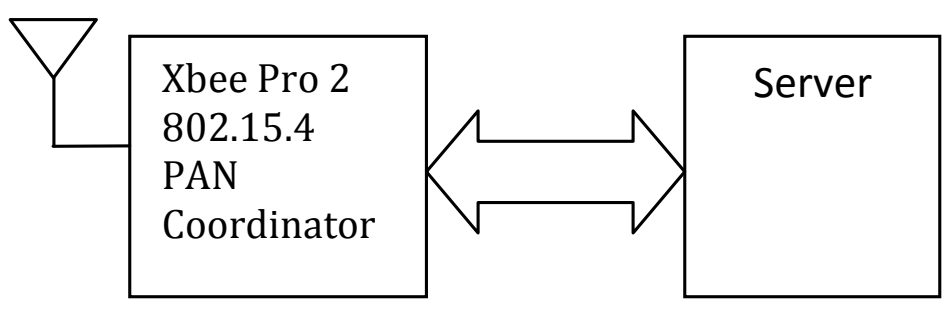

Figure 2. PAN Coordinator system with Xbee 802.15.4 series pro 2 


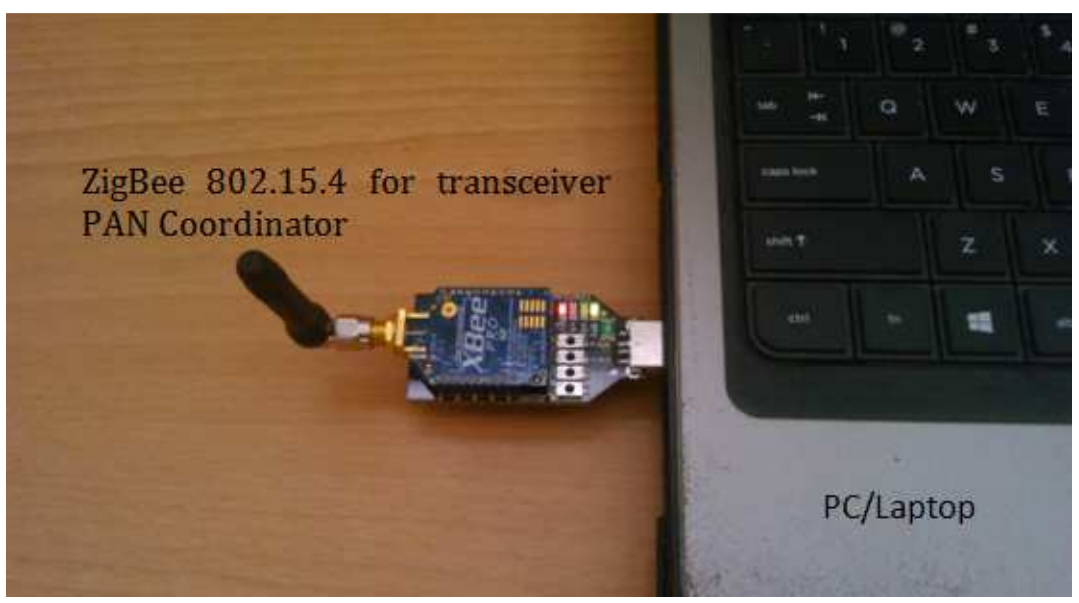

Figure 3. The hardware of the PAN coordinator with server

Figure 3 describes the hardware of the PAN coordinator with server. PAN coordinator receives data from the end device and sends to the server through a serial cable to be processed.PAN coordinator and end devices haveXBee 802.15.4 pro 2 for communications as a tranceiver. XBee 802.15.4 pro 2 has performance specifications shown in Table 1.There are maximum data rate $250 \mathrm{Kbps}$ with frequency band $2.4 \mathrm{GHz}$, Indoor range $90 \mathrm{~m}$, transmit power63 mW, supply voltage 2.7 to $3.6 \mathrm{~V}$, transmit current $120 \mathrm{~mA}$ at 3.3 VDC.

Table 1. Specification Xbee pro 2 from digi international

\begin{tabular}{|l|l|}
\hline \multicolumn{2}{|c|}{ Specification Xbee pro 2 } \\
\hline Performance & RF 250 Kbps \\
\hline Data Rate & $300 \mathrm{ft}(90 \mathrm{~m})$ \\
\hline Indoor/urban range & $2 \mathrm{miles}(3200 \mathrm{~m})$ \\
\hline Outdoor / RF Line of sight range & $63 \mathrm{~mW}(+18 \mathrm{dBm})$ \\
\hline Transmit Power & \\
\hline Feature & $2.4 \mathrm{GHz}$ \\
\hline Frequency Band & $10 \mathrm{bit}$ ADC inputs \\
\hline ADC Inputs & \\
\hline Power Requirments & 2.7 to 3.6 V \\
\hline Supplay Voltage & $120 \mathrm{~mA} @ 3.3 \mathrm{VDC}$ \\
\hline Transmit Current & $1.5 \mu \mathrm{A} @ 25$ Celcius \\
\hline Power Down Current &
\end{tabular}




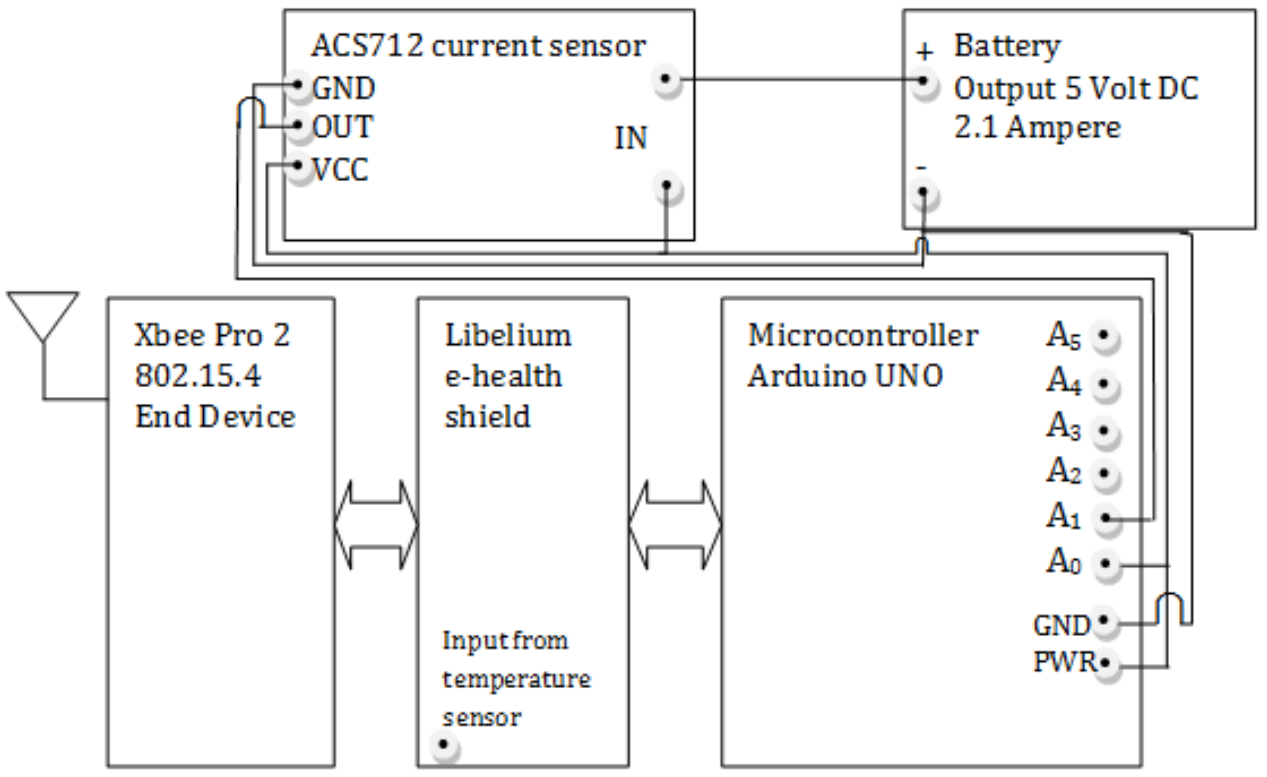

Figure 4. End Device System with Xbee 802.15.4 Pro series 2.

Figure 4 describes the system design of end device.It consists of battery for main power, ACS712 current sensor, Microcontroller arduinouno, e-health shield, and transceiver Xbee 802.15.4 pro 2.

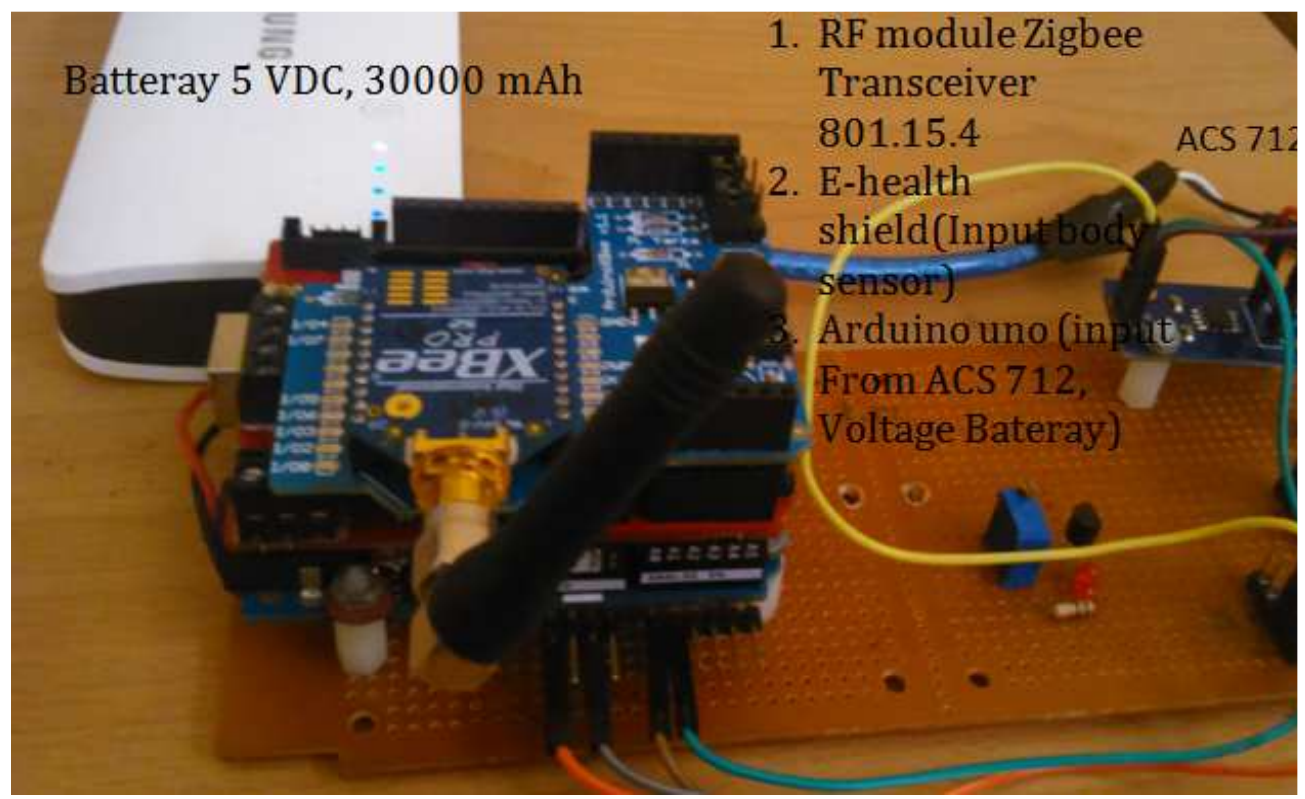

Figure 5. The hardware of the end device system with batteray

Figure 5 describes the hardware of end device system. The end device consists of Xbee 802.15.4 pro 2 RF modules, MCU, e-health shield, and baterry for main power. Baterry has a voltage of 5 volts and a current of 
$30000 \mathrm{mAh}, \mathrm{ACS} 712$ current sensors for measuring electrical currents, ehealth shield to collect data on human health, the MCU to process the data, and pro 2 XBee transceiver for communication between end devices and the PAN coordinator.

Time Scheduling algorithm system which is applied to the XBee802.15.4 pro 2 uses two different time periods: wake up and sleep period. Wake up period can be identified as TBS, while sleep period can be identified as CSP. Figure6 illustrates the TBS and CSP as period time (t) between PAN Coordinator and End Device.

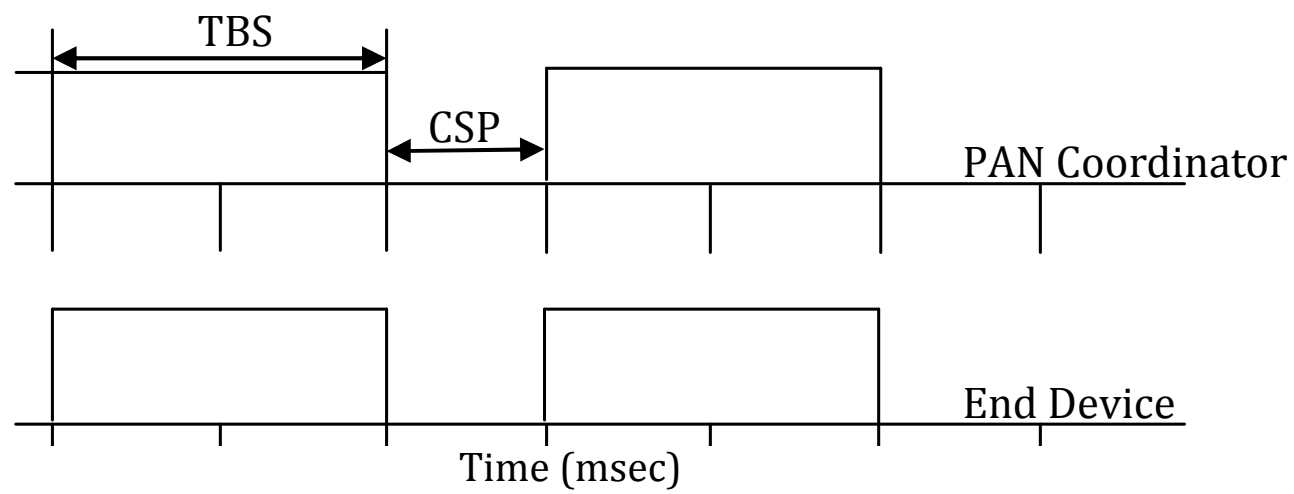

Figure 6. Diagram TBS and CSP on PAN Coordinator and End Device

MCU unit has internal Analog to Digital Converter (ADC) to convert the analog signals to digital signals in the voltage (V) and current (I) measurement using the equation (1) and (2) [16]:

$$
\begin{aligned}
& \mathrm{V}=\sum_{i=0}^{1023} \mathrm{Ui} \text { Volt } \\
& \mathrm{I}=\sum_{i=0}^{1023} \frac{(0.00489 \times \mathrm{Ui})-2.5}{0.185} \quad \text { Ampere }
\end{aligned}
$$

The ability of microcontroller arduinounoto convert data from analog to digital is 10 bits,in whichUi is analog read (i) from input analog $A_{0}$ to $A_{5}$, so that the data are generated by 1023 conditions. Each condition represents 0.00489 Volt, as a result of the division maximum input voltage $\mathrm{V}=5$ Volt by 1023 conditions. Electrical energy (E) is a multiplication between voltage (V) and current (I) as shown in equation (3) [17]:

$$
\mathrm{E}=\sum_{i=0}^{1023} \mathrm{Ui} \times \sum_{i=0}^{1023} \frac{(0.00489 \times \mathrm{Ui})-2.5}{0.185} \text { Watt }
$$




\section{EXPERIMENT AND ANALYSIS}

This experiment, CSP and DCM, will be compared by using 3 scenarios to analize the energy consumption. In this section, we will compare proposed CSP, DCM, and without schedulling (non CSP).

\subsection{Scenario 1}

In scenario 1, the determination of the active and inactive value periods based on the value of DCM is performed. DCM has two periods, namely active period and sleep period. Active period and sleep period can be determined based on the value of DCM. DCM ranges from 0.2 to 0.5 and The DCM equation is shown in (4) [11],

$$
D C M=\frac{\text { duration active period }}{\text { duration active period }+ \text { duration sleep period }} * 100 \%
$$

The non activeperiod on the Xbee802.15.4 pro 2 has 2800 msec maximum of value.The value created for non active period remains $2800 \mathrm{msec}$, while the active period is relatively determined based on the value of DCM. All parameters of the initial data for DCM methods are listed in Table 2.

Table 2.DCM parameters value at the End Device and PAN coordinator

\begin{tabular}{|r|r|r|r|r|}
\hline No. & $\begin{array}{c}\text { Value } \\
\text { DCM }\end{array}$ & \multicolumn{1}{|c|}{$\begin{array}{c}\text { Active period } \\
(\mathrm{msec})\end{array}$} & $\begin{array}{c}\text { Non active } \\
\text { period }(\mathrm{msec})\end{array}$ & $\begin{array}{c}\text { Total superframe } \\
\text { duration }(\mathrm{msec})\end{array}$ \\
\hline 1 & 0.2 & 700 & 2800 & 3500 \\
\hline 2 & 0.3 & 1200 & 2800 & 4000 \\
\hline 3 & 0.4 & 1866 & 2800 & 4666 \\
\hline 4 & 0.5 & 2800 & 2800 & 5600 \\
\hline
\end{tabular}

Table 3 describes the data used for the proposed method. TBS value at a specified scenario remains $5000 \mathrm{msec}$. CSP value is relatively determined from CSP 1000 to CSP2800 msec. CSP 2800 is the maximum value of non active period on the XBee 802.15.4 pro 2. Non CSP method does not use time scheduling; it has an active period only.

Table 3. CSP, and TBS parameters value at the End Device and PAN Coordinator

\begin{tabular}{|l|l|l|l|l|}
\hline \multirow{2}{*}{ No. } & \multicolumn{4}{|c|}{ Proposed method CSP } \\
\cline { 2 - 5 } & \multicolumn{2}{|c|}{ End device (msec) } & \multicolumn{1}{c|}{ PAN coordinator (msec) } \\
\hline & CSP & TBS & CSP & TBS \\
\hline 1 & 1000 & 5000 & 1000 & 5000 \\
\hline 2 & 2000 & 5000 & 2000 & 5000 \\
\hline 3 & 2500 & 5000 & 2500 & 5000 \\
\hline 4 & 2800 & 5000 & 2800 & 5000 \\
\hline
\end{tabular}




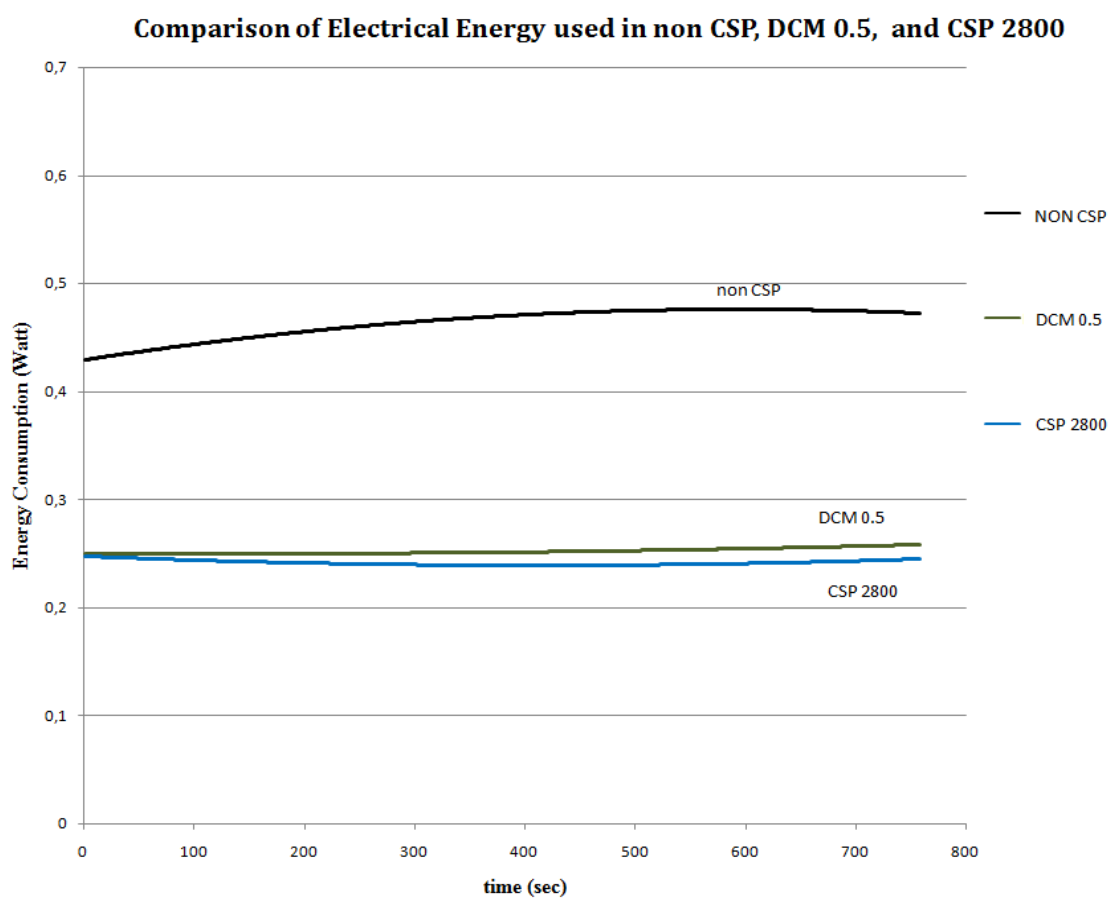

Figure 7. Energy consumption for non CSP,DCM 0.5, and CSP 2800

Figure 7 shows the graphof energy consumption amongnon CSP, DCM 0.5 and CSP 2800. The average of energy consumption of non CSP is $0.4630 \mathrm{Watt}$, DCM 0.5 is 0.2518 Watt, and CSP 2800 is 0.2413 Watt.

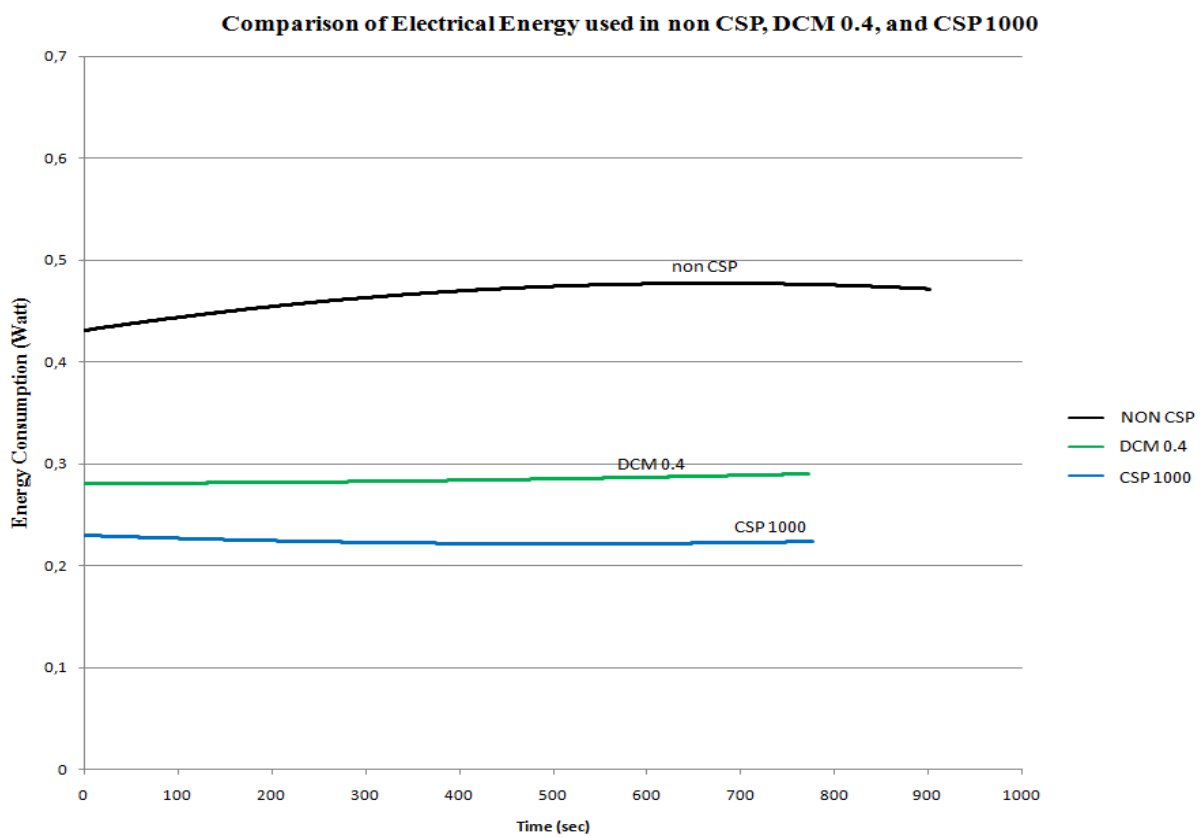

Figure 8. Energy consumption for non CSP,DCM 0.4, and CSP 1000 
Figure 8shows the graph of energy consumption amongnon CSP, DCM 0.4, and CSP 1000. The average of energy consumption of non CSP is $0.4630 \mathrm{Watt}$, DCM 0.4 is 0.2839 Watt, and CSP 1000 is0.2235 Watt.

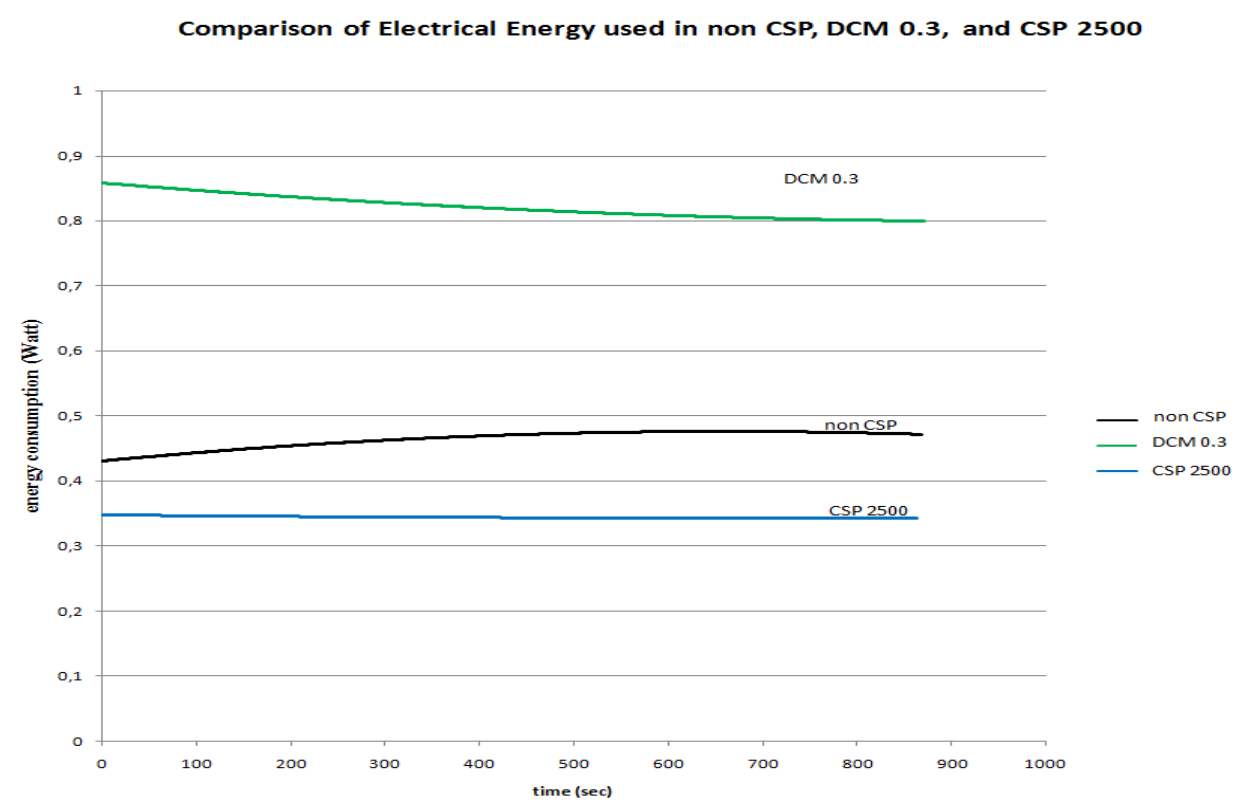

Figure 9. Energy consumption fornon CSP,DCM 0.3, and CSP 2500

Figure 9 shows the graph of energy consumption among non CSP, DCM 0.3, and CSP 2500. The average of energy consumption of non CSP is $0.4630 \mathrm{Watt}$, DCM 0.3 is 0.8213 Watt, and CSP 2500 is 0.3442 Watt.

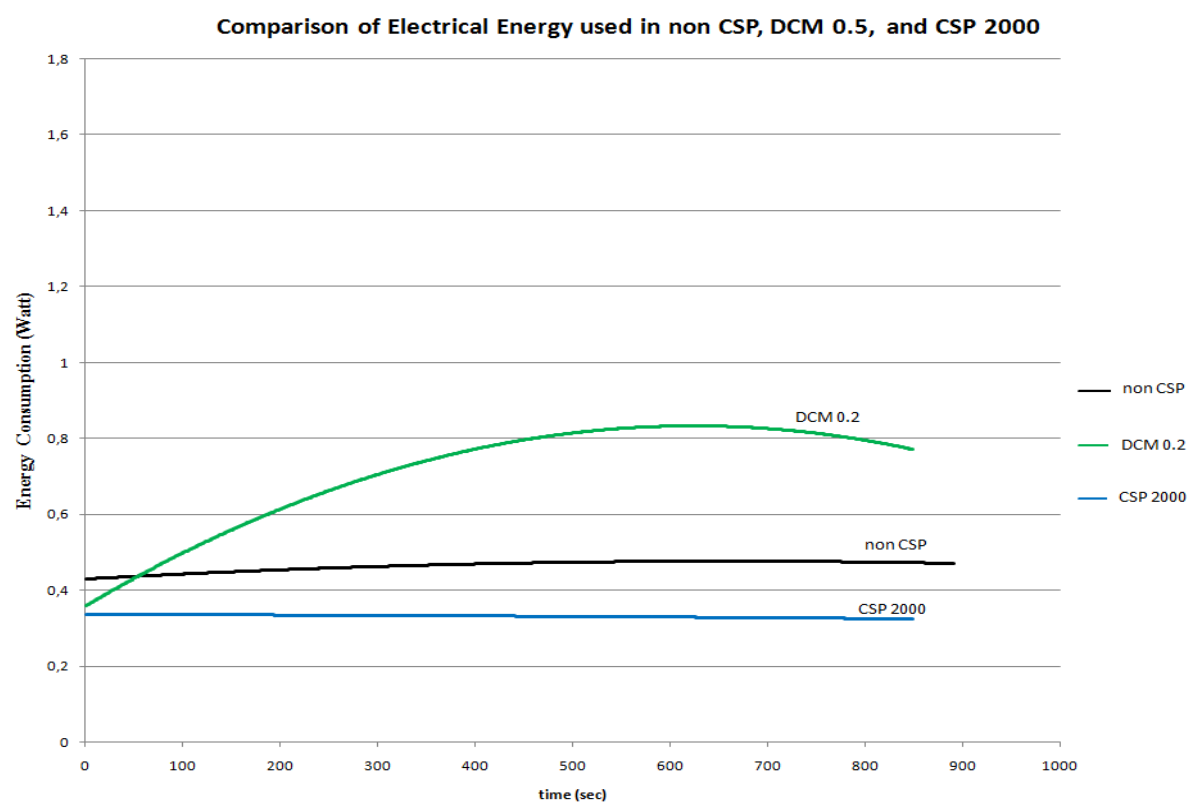

Figure 10. Energy consumption for non CSP,DCM 0.2, and CSP 2000 
Figure 10 shows the graph of energy consumption amongnon CSP, DCM 0.2 , and CSP 2000. The average of energy consumption of non CSP is $0.4630 \mathrm{Watt}$, DCM 0.2is 0.7127 Watt,andCSP 2000is0.3309 Watt.

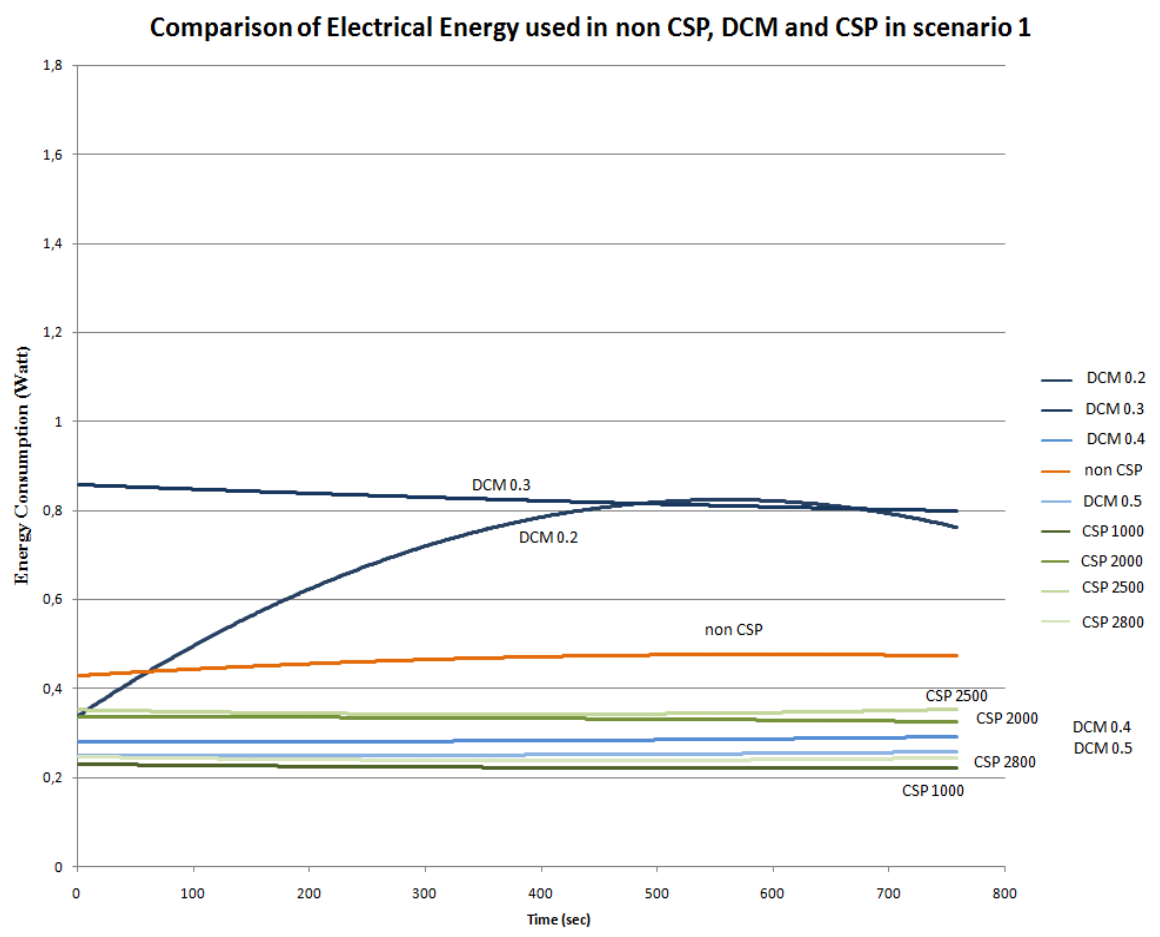

Figure 11. Energy consumption for non CSP,DCM, and CSP in scenario 1

Figure 11 shows the graph of energy consumption amongnon CSP, DCM, and CSP. The energy consumption of CSP islower than the energy consumption of non CSP and DCM in scenario 1.

\subsection{Scenario 2}

The total superframe duration in scenario 2 has a fixed value of 5600 msec. The ratio of active and non active period is captured by DCM which hasa range from 0.5 to 1 . Based on equation (4), the active and non active period values can be calculated by using equation (5), and the result is shown in table 4.

$$
\text { TotalSuperframeDuration }=\text { activeperiod }+ \text { nonactiveperiod }
$$

The parameter values in table 4 arethen used to conduct the experiment in scenario 2.It is noted in table 4 that the maximum value of non active period is $2800 \mathrm{msec}$. This is due to the maximum specification of Xbee 802.15.4 pro 2 non active period. 
Table 4. The value of the variable in DCM method fixed superframe

\begin{tabular}{|r|r|r|r|}
\hline Active (msec) & non active (msec) & $\begin{array}{c}\text { Total Superframe } \\
\text { Duration (msec) }\end{array}$ & DCM \\
\hline 5600 & 0 & 5600 & 1 \\
\hline 5040 & 560 & 5600 & 0,9 \\
\hline 4480 & 1120 & 5600 & 0,8 \\
\hline 3920 & 1680 & 5600 & 0,7 \\
\hline 3360 & 2240 & 5600 & 0,6 \\
\hline 2800 & 2800 & 5600 & 0,5 \\
\hline
\end{tabular}

The result of this scenario is shown in Figure 12. The energy consumption is compared amongthe non CSP, DCM,and the proposed CSP. The energy consumption of CSP is lesser than that of DCM and non CSP in scenario 2 .

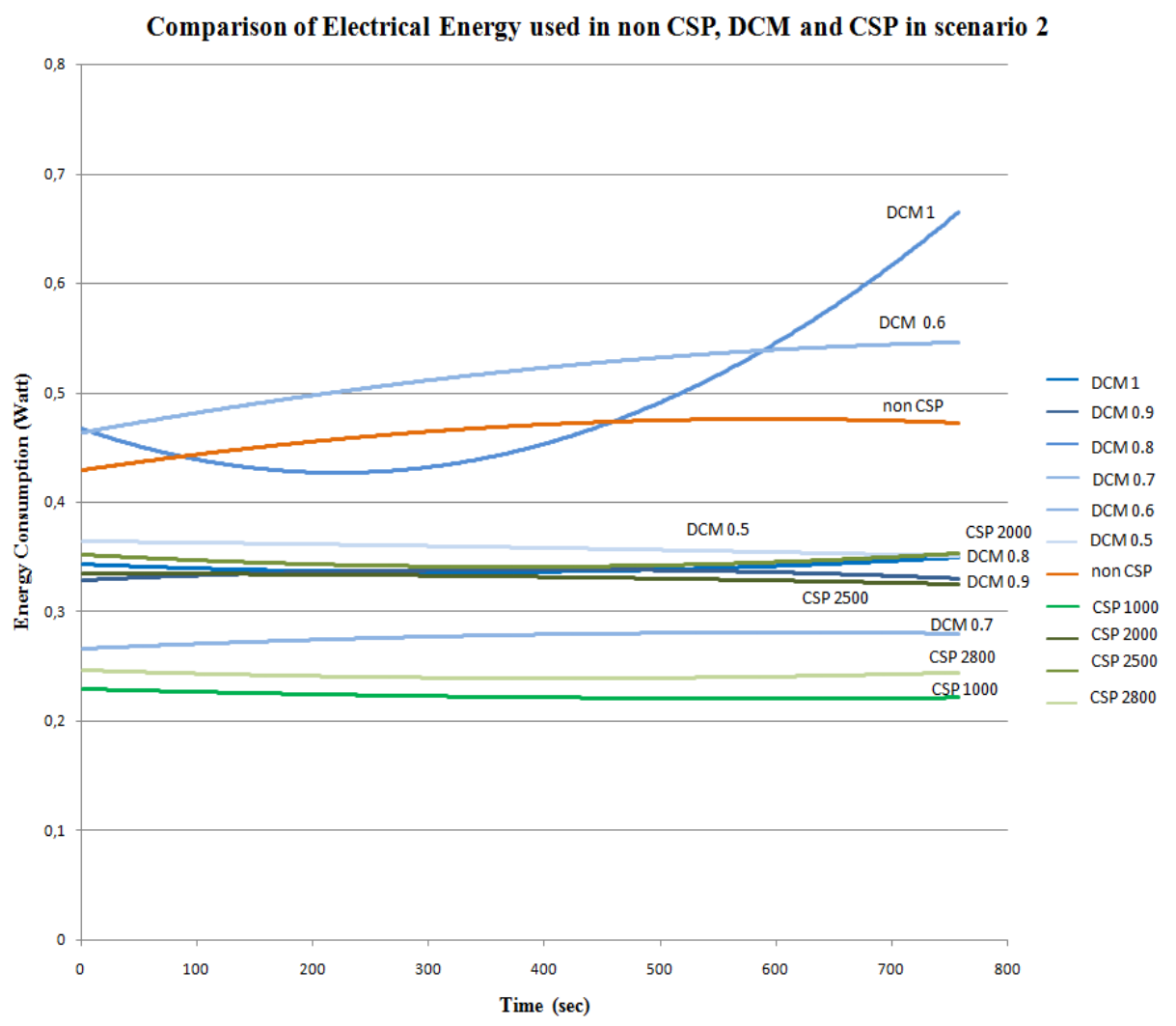

Figure 12. Energy consumption fornon CSP,DCM and CSP

\subsection{Scenario 3}

DCM method implemented in Scenario 3 has a fixed active period. The ratio of total superframe and non active periods is captured by DCM which has a range from 0.7 to 1 .Active and total superframe period valueswhich are 
obtained from the equation (4)are shown in Table 5 and the active period is computed equation (6),

$$
\text { active period }=\text { superframe duration }- \text { non active period }
$$

The parameter values in table 5 are then used to conduct the experiment in scenario 3.

Tabel 5. Scenario 3 DCM method with the value of fixed active period

\begin{tabular}{|r|r|r|r|}
\hline active (msec) & $\begin{array}{c}\text { non active } \\
(\mathrm{msec})\end{array}$ & \multicolumn{1}{|c|}{$\begin{array}{c}\text { total superframe } \\
(\mathrm{msec})\end{array}$} & DCM \\
\hline 5000 & 0 & 5000 & 1 \\
\hline 5000 & 556 & 5556 & 0.9 \\
\hline 5000 & 1250 & 6250 & 0.8 \\
\hline 5000 & 2143 & 7143 & 0.7 \\
\hline
\end{tabular}

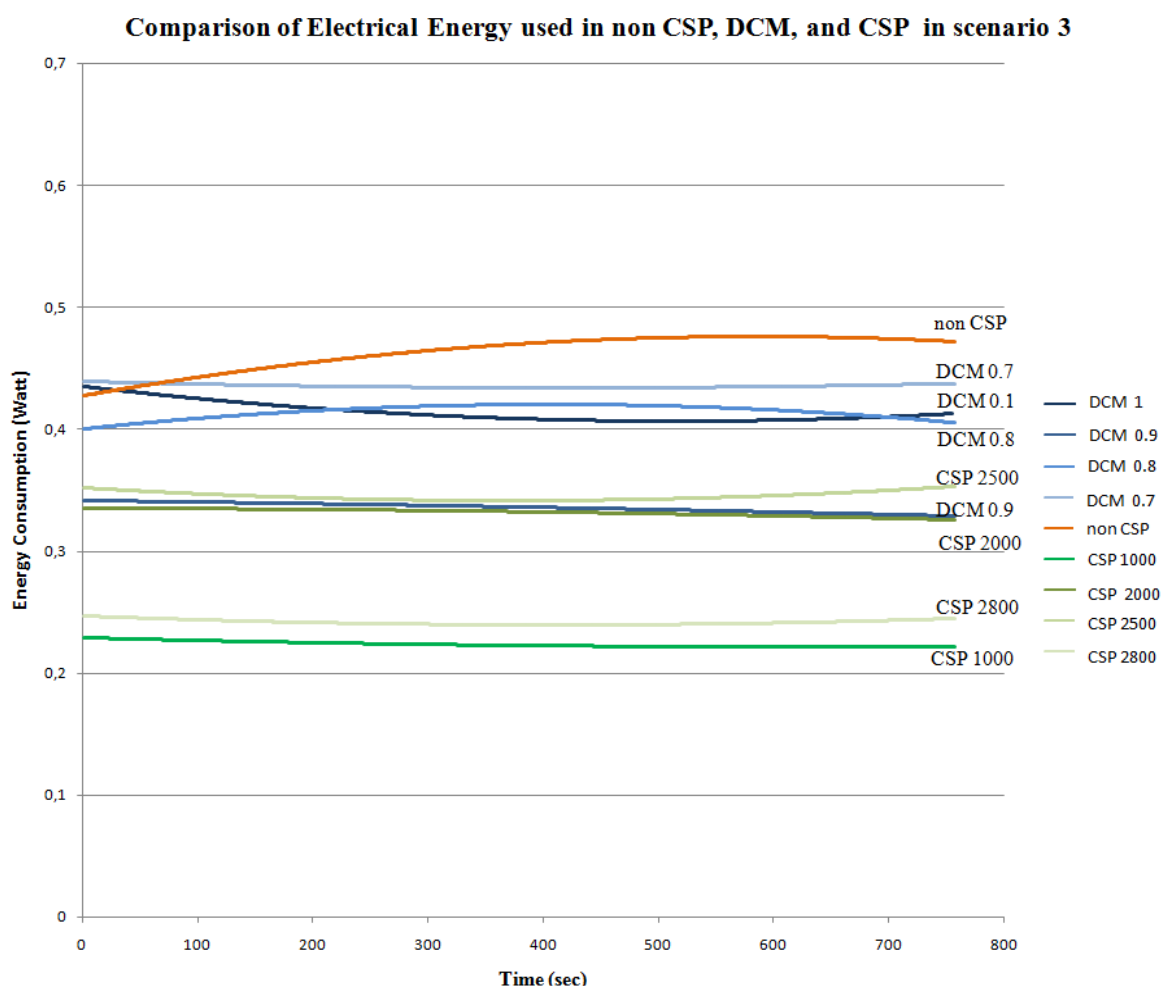

Figure 13. Energy consumption for non CSP,DCM and CSP

The result of this scenario is shown in Figure 13. The energy consumption is compared among the non CSP, DCM,and the proposed CSP. The energy consumption of CSP is lesser than that of DCM and non CSP in scenario 3. 


\section{CONCLUSION}

In this paper, we have proposed time scheduling for WBAN. Time scheduling method on WBAN is to determine the period of TBS and the CSP. Determination of TBS and the CSP period in PAN coordinator and end devices must be synchronized so that the purpose of the method of time scheduling on WBAN reached. The goals of time scheduling method are to decreaseenergy consumption. Experiments and analysis results indicate that the proposed method of time scheduling on WBAN is able to reduce the energy consumption from the three scenarios experiments, the energy consumption of CSP method is lesser than the energy consumption of DCM and non CSP method. In this experiments, we only use body temperature sensor for data transmission in the future, we will include more e-health sensor devices such as pulseoximeter sensor to evaluate the CSP method.

\section{Acknowledgements}

This research was supported in part by Ministry of Research, Technology and Higher Education of Indonesia, International Research Collaboration and Scientific Publication Scheme, under Grant No. 0094/E5.1/PE/2015.

\section{REFERENCES}

[1] IEEE 802.15.4, Part 15.4: Wireless Medium Access Control (MAC) and Physical Layer (PHY) Specifications for Low-Rate Wireless Personal Area Networks (LR-WPANs),IEEE standard for information technology, September 2006.

[2] K. Gill, S. H. Yang, F. Yao, and X. Lu, A Zigbee-based Home Automation System, IEEE Trans. Consum. Electro. vol. 55, no. 2, pp. 422-430, May 2009.

[3] M. Bertocco, G. Gamba, A. Sona, and S. Vitturi,Experimental Characterization of Wireless Sensor Networks For Industrial Applications, IEEE Trans. Instrum. Meas., vol. 57, no. 8, pp. 1537-1546, August 2008.

[4] Y. Kim, R. G. Evans, and W. M. Iversen, Remote Sensing and Control of an Irrigation System using a Distributed Wireless Sensor Network, IEEE Trans. instrum. Meas., vol. 57, no. 7, pp. 1379-1387, July 2008.

[5] S. H. Lee, S. Lee, H. Song, and H. S. Lee, Wireless Sensor Network Design for Tactical Military Applications: Remote Large-scale Environments, in Proc. MILCOM, October 2009.

[6] M.U.H. Al Rasyid, B-H. Lee, A. Sudarsono, I. Mahfud, "Monitoring System of Patient Position Based On Wireless Body Area Sensor Network", IEEE International Conference on Consumer Electronics Taiwan (ICCE-TW), June 6-8, 2015. 
[7] K.S. Kwak, M. A. Ameen, C. Lee, H. Lee, A Study on Proposed IEEE 802.15 WBAN MAC Protocols, The International Symposium on Communications and Information Technologies, ISCIT September 28-30, 2009.

[8] W. Weiya, L. Chao, Integrated Application of WBAN and WSN, Fifth International Joint Conference on INC, IMS and IDC, 2009.

[9] B-H. Lee, M.U.H. Al Rasyid, H-K. Wu, Analysis of Superframe Adjustment and Beacon Transmission for IEEE 802.15.4 Cluster Tree Networks, EURASIP Journal on Wireless Communications and Networking, July 2012.

[10] J. Kim, I. Song, E. Jang, S. Choi, A Dynamic Duty Cycle MAC Algorithm for Wireless Body Area Networks, International Journal of Bio-Science and Bio-Technology, Vol. 4, No. 2, June, 2012.

[11] H. Rasouli, Y.S. Kavian, H.F. Rashvand, ADCA: Adaptive Duty Cycle Algorithm for Energy Efficient IEEE 802.15.4 Beacon-Enabled Wireless Sensor Networks, IEEE Sensors Journal, pp. 3893 - 3902, 24 September 2014.

[12] K.S. Deepak, A.V. Babu, Energy EFFiciency of IEEE 802.15.6 based Wireless Body Area Networks in Scheduled Access Mode, International Conference on Advances in Computing, Communications and Informatics (ICACCI), pp301 - 307, 22-25 Aug. 2013.

[13] M.T. IshtaiqueulHuque, K.S. Munasinghe, M. Abolhasan, A. Jamalipour, EAR-BAN: Energy Efficient Adaptive Routing in Wireless Body Area Networks, 7th International Conference on Signal Processing and Communication Systems (ICSPCS), pp. 1 - 10, 16-18 Dec. 2013.

[14] H. Karvonen, J. Petäjäjärvi, J. Iinatti, M. Hämäläinen, C. Pomalaza-Ráez, A Generic Wake-Up Radio Based MAC Protocol for Energy Efficient Short Range Communication, Proc. PIMRC, 2014.

[15] J. Petajajarvi, H. Karnoven, K. Mikhylov, A. Parssinen, M. Hamalainen, J. Iinatti, WBAN Energy Efficiency and Dependability Improvement Utilizing Wake-Up Receiver, IEEE Trans. Commun, April 2015.

[16] 0. O. Olakanmi, O. M. Ojone, A. O. Seun, I. D.Adeyemi, A.K.Joseph,T. A.Taofeek, 0. O. Abel, and A. A.Rasaki, A Microcontroller-based Design for Energy Efficient Nickel-chrome Plating Process, American Chemical Science Journal,2015.

[17] G. Rostirolla, R.D. R. Righi, V. F. Rodrigues, P. Velho, E. L.Padoin, GreenHPC: A Novel Framework to Measure Energy Consumption on HPC Applications, International Federation for Information Processing (IFIP), 2015. 